\title{
Carcinoma adenoide cístico intracraniano
}

\author{
Leonardo C. Welling ${ }^{1}$, José Carlos Lynch², Ricardo Andrade ${ }^{3}$, \\ Celestino Esteves Pereira ${ }^{3}$, Fabiana Hidalgo ${ }^{1}$, Juliano Corrêa ${ }^{1}$, Lúcia Kaskus ${ }^{4}$
}

Serviço de Neurocirurgia do Hospital dos Servidores do Estado do Rio de Janeiro (HSE/RJ). Serviço de Anatomia Patológica do HSE/RJ.

\section{RESUMO}

Os carcinomas adenoides císticos são tumores de crescimento lento, localmente agressivos e com tendência à recorrência. Têm particular interesse por infiltrar estruturas neurais e disseminar-se pelo perineuro. Envolvimento intracraniano é raro. Descreve-se um caso de carcinoma adenoide cístico originado na glândula lacrimal esquerda e invasão do seio cavernoso. Ressonância magnética demonstrou lesão tumoral, extra-axial, bem delimitada, semelhante a um meningioma da asa do esfenoide com invasão do seio cavernoso. O paciente apresentou boa resposta ao tratamento com cirurgia e radioterapia complementar.

\section{PALAVRAS-CHAVE}

Carcinoma, intracraniano.

\begin{abstract}
Intracranial adenoid cystic carcinoma

Adenoid cystic carcinoma is a relatively common head and neck tumor that is slow growing, but locally aggressive and thus prone to recurrence. It is of particular interest because of its tendency to locally infiltrate neural structures and to spread perineurally. Intracranial involvement has been regarded as rare. A case report of a patient with adenoid cystic carcinoma originated in the left lacrimal gland and involving the cavernous sinus is presented. Magnetic resonance image demonstrated a welldemarcated enhancing extra-axial tumour, resembling a sphenoid ridge meningioma extending into the cavernous sinus. This patient showed a good response to treatment with surgery followed by post-operative radiotherapy
\end{abstract}

\section{KEYWORDS}

Carcinoma, intracranial.

\section{Introdução}

O carcinoma adenoide cístico (CAC), uma neoplasia epitelial maligna, foi descrito pela primeira vez em 1856. ${ }^{9}$ A maioria dos CAC origina-se das glândulas salivares maiores e menores, nasofaringe e glândulas lacrimais. ${ }^{4,6}$ É reconhecido pelo seu crescimento lento, padrão de recorrência e metástases a distância. Corresponde a apenas $1 \%$ das neoplasias de cabeça e pescoço e a invasão intracraniana ocorre em cerca de $4 \%$ dos casos. ${ }^{2}$

Neste artigo, descreve-se um caso de carcinoma adenoide cístico originado na glândula lacrimal esquerda com invasão das estruturas intracranianas através de fissura orbital superior e seio cavernoso.

\section{Relato do caso}

Paciente masculino, 46 anos, com história de cefaleia e ptose palpebral com quatro anos de evolução. $\mathrm{Na}$ admissão, apresentava oftalmoplegia completa e amaurose à esquerda. Ainda durante a internação, apresentou quadro de nevralgia trigeminal comprometendo os territórios de V1 e V2. Ressonância magnética de encéfalo evidenciou lesão expansiva com captação intensa de contraste localizado na órbita esquerda, em situação retro-orbitária intra e extraconal, estendendo-se ao ápice orbitário, fissura orbitária superior, seio cavernoso (envolvendo a artéria carótida), cavum de Meckel e forâmen redondo e oval (Figuras 1A, 1B e 1C). Foi submetido ao tratamento cirúrgico, craniotomia pterional

1. Médico-residente do Serviço de Neurocirurgia do Hospital Servidores do Estado do Rio de Janeiro (HSE/RJ).

2. Neurocirurgião, chefe do Serviço de Neurocirurgia do HSE/RJ.

3. Neurocirurgião do HSE/RJ.

4. Médica patologista do Serviço de Anatomia Patológica do HSE/RJ. 
esquerda, com remoção parcial da lesão em virtude das estruturas envolvidas (Figura 1D). No pós-operatório, apresentou melhora do quadro de nevralgia.

Análise histopatológica evidenciou estruturas cribiformes e alguns túbulos formados por células basaloides com pleomorfismo nuclear discreto e raras mitoses (Figura 1E). Encaminhado à radioterapia como terapia complementar e permanece em acompanhamento ambulatorial até a presente data.

\section{Discussão}

Os carcinomas adenoides císticos são neoplasias epiteliais que se originam nas glândulas salivares maiores, menores, nasofaringe e glândulas lacrimais. Histopatologicamente, o CAC pode expressar-se de forma variável, sendo reconhecidos três padrões principais. ${ }^{3}$ O padrão cribriforme, o mais clássico, caracteriza-se por ilhas de células epiteliais basaloides, pequenas ou cuboidais, exibindo núcleo extremamente basofílico, com pouco citoplasma e múltiplos espaços císticos. ${ }^{3}$ No padrão tubular, as células epiteliais estão arranjadas em múltiplos ductos, pequenos ou túbulos, envolvidos no estroma hialino e na variante sólida, ilhas ou lençóis que demonstram pouca tendência a formar ductos ou espaços císticos. ${ }^{3}$ Diferentemente do padrão cribriforme e tubular, no padrão sólido, pode-se observar pleomorfismo celular e atividade mitótica, bem como focos de necrose nos centros das ilhas das células neoplásicas. Desses, o padrão sólido é o com pior prognóstico. ${ }^{5}$

A invasão intracraniana do carcinoma adenoide cístico ocorre, principalmente, através das estruturas perineurais. Porém, há relatos de invasão direta e disseminação hematogênica. ${ }^{10}$

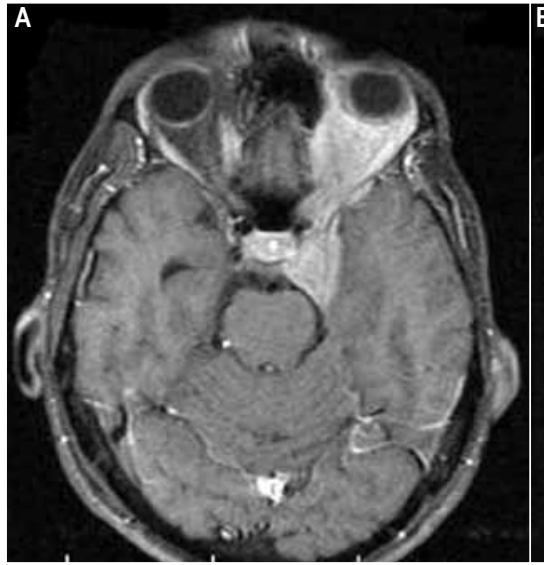

Figura $1 A$ - RM corte axial: lesão com captação de contraste localizado na órbita esquerda, em situação retro-orbitária com extensão intracraniana.

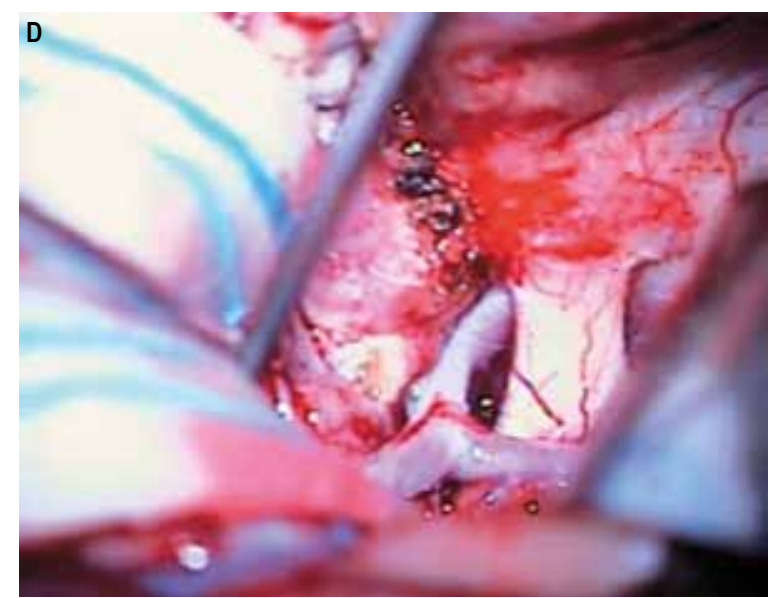

Figura $1 D$ - Observa-se a lesão tumoral lateralmente à artéria carótida interna esquerda, sua bifurcação e o nervo óptico.

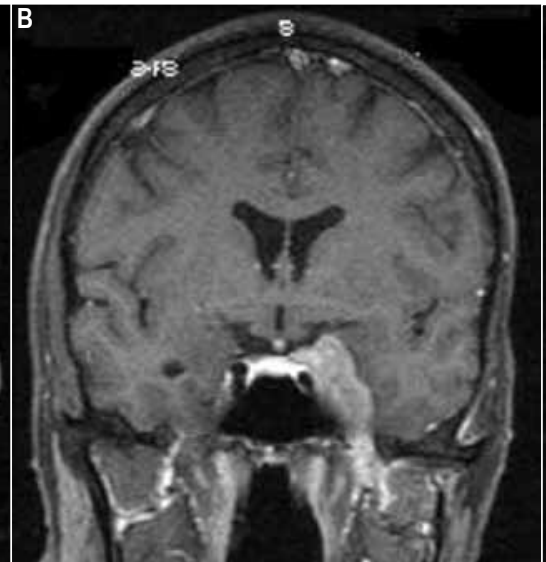

Figura 1B - RM corte coronal.

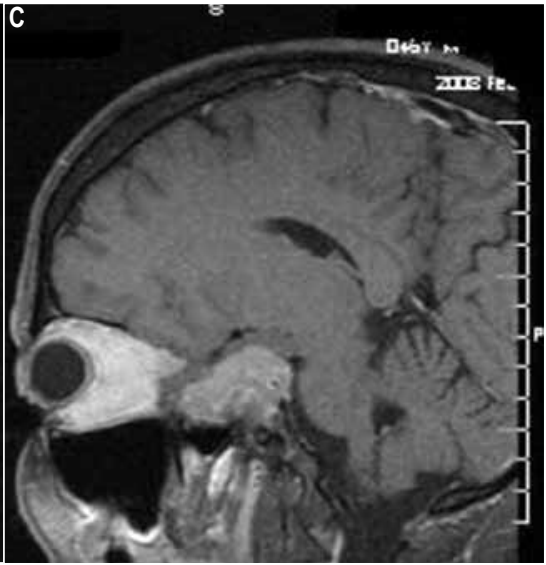

Figura 1C-RM corte sagital.

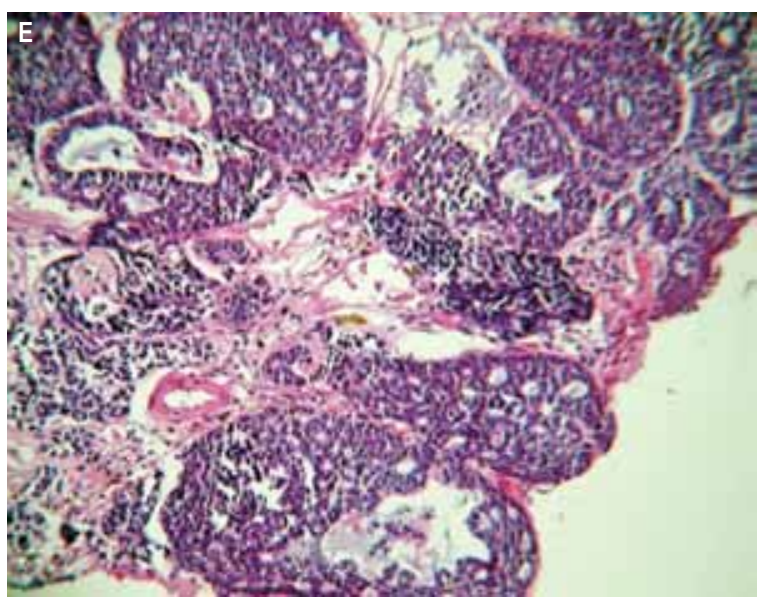

Figura 1E - Coloração HE. Notam-se estruturas cribiformes e alguns túbulos, formados por células basaloides com pleomorfismo nuclear discreto e raras mitoses. 
Cerca de $40 \%$ dos carcinomas adenoides císticos recorrem em até três anos, mesmo nos totalmente ressecados. ${ }^{1}$ Se o controle local for alcançado, metástases a distância ocorrem em até $39 \%$ dos casos, principalmente para os pulmões, ossos e fígado.

Apesar de o tratamento cirúrgico seguido de radioterapia complementar ser a abordagem de escolha nos pacientes com tumores malignos das glândulas salivares, nos casos em que ocorre a invasão intracraniana, a sobrevida livre de doença é menor, muitas vezes pelo fato de raramente se conseguir a remoção total da lesão. ${ }^{8} \mathrm{De}$ modo geral, o prognóstico permanece reservado com sobrevida de $50 \%$ em cinco anos. ${ }^{7}$

\section{Conclusão}

O carcinoma adenoide cístico é uma patologia com prognóstico ruim e pouco reconhecida. A remoção cirúrgica seguida de radioterapia complementar é o atual tratamento de escolha. Novos estudos são necessários para avaliar a eficácia da radiocirurgia na terapêutica dessa afecção.

\section{Referências}

1. Adachi K, Yoshida K, Ueda R, Kawase T. Adenoid cystic carcinoma of the cavernous region: case report. Neurol Med Chir (Tokyo). 2006;46:358-60.
2. Alleyne $\mathrm{CH}$, Bakay RE, Costigan D, Thomas B, Joseph GJ. Intracranial adenoid cystic carcinoma: case report and review of the literature. Surg Neurol. 1996;45:265-71.

3. Altman KW. Pathologic quiz case. Adenoid cystic carcinoma of parotid gland. Arch Otolaryngol Head Neck Surg. 1997;123(12):1352-55.

4. Benazzou S, Arkha Y, Boulaadas M, Derraz S, Essakali L, Kzadri M. Nasal adenoid cystic carcinoma with intracranial invasion. J Craniofac Surg. 2006;17(5):1026-9.

5. Bradley PJ. Adenoid cystic carcinoma of the head and neck: a review. Curr Opin Otolaryngol Head Neck Surg. 2004;12:127-32.

6. Gormley WB, Shekhar LN, Wright DC, Olding M, Janecka IP, Snyderman $\mathrm{CH}$, et al. Management and long-term outcome of adenoid cystic carcinoma with intracranial extension: a neurosurgical perspective. Neurosurgery. 1996;38:1105-13.

7. Meldrun ML, Tse DT, Benedetto P. Neoadjuvant intracarotid chemotherapy for treatment of advanced adenocystic carcinoma of lacrimal gland. Arch Ophtalmol. 1998;116:315-21.

8. Pitman KT, Prokopakis EP, Aydogan B. The role of skull base surgery for the treatment of adenoid cystic carcinomam of the sinonasal tract. Head Neck. 1999;21:402-7.

9. Terasaki M, Tokutomi $\mathrm{T}$, Maruiwa $\mathrm{H}$, Sugita $\mathrm{Y}$, Harada $\mathrm{H}$, Shigemori M. High-grade adenoid cystic carcinoma originating from the lacrimal gland. Brain Tumor Pathol. 2000;17:159-63.

10. Tsuyuguchi N, Ohata K, Goto T, Haque M, Hara M. Intracranial adenoid cystic carcinoma of suprasellar region. Acta Neurochir (Wien). 2001;143:729-32.

Endereço para correspondência

Leonardo C. Welling

Rua Tiradentes, 976, sala 1, Centro

84010-190 - Ponta Grossa - PR

E-mail: leonardowelling@yahoo.com.br 